\title{
Uterine Tachysystole, Hypertonus and Hyperstimulation: An urgent need to get the definitions right to avoid Intrapartum Hypoxic- Ischaemic Brain Injury
}

\author{
Suganya Sukumaran1, Yan-Ju Jia ${ }^{2}$ and Edwin Chandraharan ${ }^{3 *}$ \\ ${ }^{1}$ Obstetrics \& Gynaecology, South Warwickshire NHS Foundation Trust, UK \\ ${ }_{2}^{2}$ Tianjin Central Hospital of Obstetrics and Gynaecology, Tianjin Key Laboratory of Human Development and Reproductive Regulation, China \\ ${ }^{3}$ Global Academy of Medical Education \& Training, London \& Consultant Intrapartum Care Advisor, Basildon University Hospital, UK
}

Submission: April 17, 2020; Published: April 30, 2020

*Corresponding author: Edwin Chandraharan, Global Academy of Medical Education \& Training, Consultant Intrapartum Care Advisor, UK

\begin{abstract}
Onset of labour is due to the hormonal changes initiated by the hypo-thalamo-pituitary axis followed by local changes in the chorio-amnion that result in the production of prostaglandins (PGs). Prostaglandins cause softening and effacement of the cervix and uterine contractions that are key elements for the onset and progress of labour. Uterine contractions have four important parameters: the frequency, strength, duration, and the basal "resting" tone, and if any of these parameters are excessive, a reduction in fetal oxygenation may occur. It is important to understand that any increase in uterine activity (frequency, strength, duration or the basal "resting" tone) can affect the utero-placental oxygenation and the patency of the blood vessels within the umbilical cord. Any disturbance in fetal oxygenation secondary to an increase in the frequency, duration and strength or the basal tone of the uterine contractions sufficient to cause fetal hypoxia can cause changes in the fetal heart rate patterns. There is a wide variation in current National and International Guidelines on what constitutes excessive uterine activity, and most guidelines do not consider the duration, strength or increased basal tone in their definition of uterine hyperstimulation. This may lead to the failure of recognition of an ongoing excessive uterine activity leading to poor maternal and perinatal outcomes.
\end{abstract}

Keywords: Uterine hyperstimulation; Cardiotocograph (CTG); Hypertonus, Tachysystole; Hypoxic ischaemic encephalopathy (HIE); Acute tocolysis

Abbreviations: CTG: Cardiotocograph; HIE: Hypoxic Ischemic Encephalopathy; ACOG: American College of Obstetricians and Gynecologists; UH: Uterine Hyperstimulation; UT: Uterine Tachysystole; RUPI: Relative Utero-Placental Insufficiency

\section{Introduction}

Uterus acts as a receptacle for the fetus from conception to until the time of birth. Progressively increasing frequency, duration and strength of uterine contractions are key to achieve progress of labor culminating in vaginal birth. Uterine contractions have four important parameters: the frequency, strength, duration, and the resting tone, and the very low resting tone is very important as this helps in ensuring fetal oxygenation in between contractions. This is because fetal oxygenation and removal of metabolic waste products occurs predominantly during periods of uterine relaxation, when the maternal spiral arterioles and uterine venules feeding the placental venous sinuses are open and the blood vessels within the umbilical cord are not compressed. At rest, the pressure within the spiral arteries is greater than the intramyometrial pressure, resulting in blood flow into the intervillous space. The waste products exit through the low-pressure venous system into the maternal circulation. During a uterine contraction, the myometrial pressure exceeds the spiral arterial pressure, and the uterine blood flow is interrupted causing a cessation in oxygen delivery to the fetus. The duration of the spiral artery compression depends on the strength and the duration of the contraction. In a recent systematic review by Reynolds et al, an increased uterine activity was found to be a non-specific predictor of depressed 


\section{Global Journal of Reproductive Medicine}

neurological function in the newborn [1]. Similarly, it has been shown that six or more contractions in 10 minutes were significantly associated with fetal heart rate decelerations ( $\mathrm{P} \leq$.001) [2]. Most of the guidelines for the CTG interpretation mention about the fetal heart rate pattern with minimal information about uterine activity. A recent survey by $\mathrm{Al}-\mathrm{Hafez}$ et al identified inconsistency in the definition, frequency with which uterine tachysystole is encountered and its hypothetical management [3]. It concluded that there was ambiguity regarding tachysystole despite American College of Obstetricians and Gynecologists (ACOG) attempts to rectify it. Hypoxemia may result if contractions are lasting longer and / or if they are stronger or are more frequent. It has been shown that a mean contraction interval of fewer than 2.3 minutes decreases the oxygenation saturation levels in the fetal brain, when oxytocin is used to augment labor [4].

\section{Definitions}

The importance of uterine contractions to the process of parturition was recognized early in obstetric practice and there have been attempts to objectively assess them for at least two centuries. Various definitions exist for uterine hyperstimulation or uterine tachysystole (UT), and therefore, the Eunice Kennedy Shriver National Institute of Child Health and Human Development (NICHD) in conjunction with ACOG and SMFM adopted a standardized definition for tachysystole: more than five contractions in a 10-minute period, averaged over a 30-minute window [5]. However, this definition does not address the other parameters such as hypertonus, duration or strength of uterine contractions. This may lead to missing an ongoing excessive uterine activity secondary to an increase in the tone or duration of uterine contractions. Several CTG guidelines have offered minimal or no explanation regarding the underlying pathophysiology of the increased uterine activity such as intra-amniotic or maternal infection or placental abruption which may have a significant impact on the mothers and neonates, but these may be missed by clinicians as they do not fulfil the criteria for "uterine hyperstimulation" as stipulated by these guidelines.

\section{Current (mis)understanding Uterine Hyperstimulation (UH)}

The correct definition should encompass a combination of frequency, duration, strength as well as the basal tone of the uterine contractions. Therefore, uterine hyperstimulation should be defined as "any increase in uterine activity associated with a CTG change". However, some guidelines (e.g., National Institute of Health and Care excellence or NICE 2017) [6] have simply used the number of uterine contractions ( $>5$ in 10 minutes) to define uterine hyperstimulation, and this over-simplified definition may lead to missing ongoing excessive uterine activity secondary to an increase in the tone or duration of uterine contractions. Such a simplified approach may lead to hypoxic ischemic encephalopathy (HIE), especially in growth restricted fetuses with poor uteroplacental reserve. A survey among the CAOG (Central Association of Obstetricians and Gynecologists) noted inconsistency in the definition, frequency with which it is encountered and hypothetical management of uterine tachysystole along with ambiguity regarding tachysystole [3] despite the attempts by the ACOG to standardize the definition. The standard assessment of the fetal wellbeing during high-risk labor is continuous electronic fetal heart rate monitoring using the Cardiotocography (CTG). As the name implies various parameters of the fetal heart rate (Cardio) and the frequency and duration of uterine activity (Toco) are recorded on the CTG trace. It has been shown that excessive uterine activity by means of uterine tachysystole, shortens the relaxation time resulting in higher levels of cerebral deoxygenated hemoglobin, lower levels of oxygenated hemoglobin and decreased intracerebral oxygen saturation [4]. A minimum inter-contraction interval of 2.3 minutes appears to be crucial in ensuring cerebral oxygenation when oxytocin is used to augment labor. Therefore, the standard definitions of uterine hyperstimulation as suggested by NICE Guidelines [6] may cause harm as it does not consider the duration, strength, or the resting tone of the uterine contractions, and therefore, they should not be used when oxytocin is used to augment labor. This is because oxytocin increases the frequency, duration and strength of the uterine contractions and reduces the relaxation time. In addition, it also increases the basal tone of the uterus leading to reduced filling time. It has been shown that even in spontaneous labor, the mean intracerebral saturation drops by $54 \%$ during contraction and in the presence of tachysystole, the oxygen saturation can be down to $18 \%$ [4]. It is also vital to understand that UH can occur in both spontaneous and induced labor.

\section{Spontaneous uterine tachysystole (UT)}

A retrospective cohort study involving 890 patients, concluded that $11 \%$ had at least one episode of UT and the newborns in the tachysystole group were two times more likely to be admitted to NICU [7]. The pathological causes for spontaneous UT are suspected chorioamnionitis (subclinical and clinical) and Antepartum hemorrhage (i.e., blood seeping in between the myometrial fibers or separating the membranes from the decidua leading to uterine irritability). It has been shown that in cases of placental abruption during the 2-h period preceding delivery the CTG showed five or more contractions in $10 \mathrm{~min}$, with a mean of 5.6 [8]. Therefore, occurrence of UT in spontaneous labor (Figure 1) should be viewed with a high index of suspicion for an ongoing chorioamnionitis or a concealed placental abruption. It is vital to appreciate that tocolysis may not be appropriate in women with UT secondary to placental abruption or chorioamnionitis, especially if there is maternal tachycardia or hypotension. If spontaneous vaginal birth is not imminent, delivery should be expedited in these cases. Tocolytics do not correct the underlying pathology in placental abruption and chorioamnionitis, and they should only be considered in the absence of maternal tachycardia or hemodynamic instability to transiently improve fetal oxygenation until an urgent delivery is accomplished to avoid fetal compromise. UT has also been reported in women who had previous caesarean section and uterine rupture in subsequent pregnancies. Based on the 


\section{Global Journal of Reproductive Medicine}

pathophysiology of complete separation of the uterine wall, such changes in uterine activity are expected, and these were found to occur in $38 \%$ of the women with uterine rupture compared to $21 \%$ in the control group [9]. Uterine tachysystole is not only associated with fetal hypoxia, but it has also been shown to be associated with brachial plexus injury in the neonates, and the odds of this complication was 3.7 times higher when tachysystole occurred [10]. In subclinical or clinical chorioamnionitis, the baseline fetal heart rate would be higher than expected for the given gestational age. Therefore, if there is UT in the CTG even with the normal fetal heart pattern, it is essential to exclude on going chorioamnionitis as the cause of UT. If the amniotic fluid is blood stained or if the patient presents with an antepartum hemorrhage associated with UT, an underlying placental abruption should be suspected and if there are associated abnormalities on the fetal heart rate, delivery should be expedited to avoid fetal compromise. Presence of "lowvoltage complexes" on the tocograph indicating myometrial irritability should be considered very seriously.

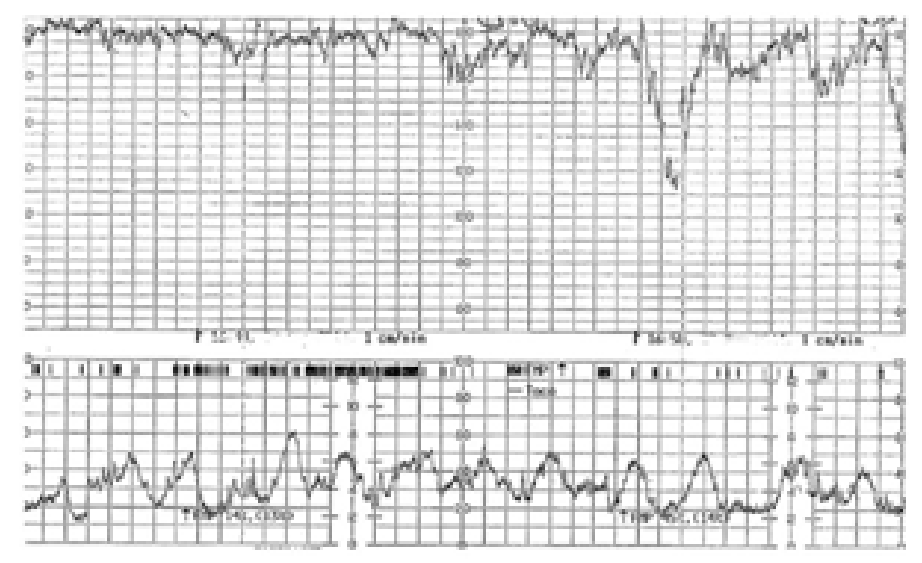

Figure 1: Spontaneous Hyperstimulation secondary to chorioamnionitis confirmed on Histology without the use of prostaglandins or oxytocin.

\section{Uterine tachysystole or UH in induced/augmented labour}

The overall rate of induction of labor in the European Union (EU) and United States (USA) is reported to be in the range of $20-25 \%$. A recent systematic review on labor induction with prostaglandins, found that the results relating to uterine hyperstimulation (Figure 3) were not always reported, and, when they were reported, UH was defined inconsistently or not all [11]. Mechanical methods such as transcervical foley catheter compared to prostaglandins appeared to show a reduced incidence of uterine tachysystole [12]. Another systematic review on methods of labor found that the risk of hyperstimulation with or without fetal heart rate changes was significantly higher in the prostaglandin group compared to the placebo [13]. However, insufficient data prohibited any conclusions about serious maternal or neonatal morbidity or death [14]. When cervical PGE2 was compared with a placebo or no treatment, there was a significant increase in hyperstimulation without fetal heart rate changes. However, the maternal and neonatal outcomes were infrequently reported, and therefore, meaningful conclusions could not be drawn regarding the detrimental effect of the hyperstimulation [15]. Compared with dinoprostone, the use of the misoprostol insert was associated with an increased likelihood of uterine tachysystole, but the rate of tachysystole requiring tocolysis was not significantly different between the 2 groups [16]. In studies comparing oxytocin with prostaglandins, no significant increase in hyperstimulation was noted in the oxytocin group with a small increase in chorioamnionitis in prostaglandin group [17]. In comparing mechanical methods such as laminaria tent, Foley's catheter, the risk of hyperstimulation was much less compare to the prostaglandin group and but infants born to mothers undergoing mechanical methods of induction were more likely to experience neonatal infectious morbidity than infants born to mothers undergoing induction with pharmacologic methods [18]. When oxytocin was used for augmentation of labor, high doses of oxytocin and the decrease in the oxytocin-dosing interval for augmentation of labor both induced uterine hyperstimulation [19]. Increased uterine activity was associated with a higher incidence of fetal heart rate abnormalities and neonatal acidosis [19].

\section{Definition of uterine hyperstimulation \& uterine hypertonus}

Some have argued that the term uterine hyperstimulation should only be reserved for an increase in uterine frequency associated with induction or augmentation of labor (i.e., due to excessive myometrial stimulation). However, such an oversimplified approach will miss fetuses exposed to increased 


\section{Global Journal of Reproductive Medicine}

strength, duration, or the basal tone of uterine contractions. Excessive uterine activity may cause variable, late or prolonged decelerations with changes in baseline fetal heart rate variability. It is vital to appreciate that in a rapidly evolving hypoxia secondary to excessive uterine activity, the baseline fetal heart rate may be increased (i.e., the "Zig Zag Pattern" pattern) due to an autonomic instability $[20,21]$. Therefore, any increase in uterine activity (i.e., frequency, duration, strength, or the basal tone) associated with abnormalities in the fetal heart rate should be considered as uterine hyperstimulation (Figure 2). Uterine hypertonus (Figure 3 ), on the other hand, should be reserved for changes in fetal heart rate associated with a sustained uterine contraction as would be seen secondary to the use of prostaglandins or oxytocin. A fetus with a relative utero-placental insufficiency (RUPI) may show an increased FHR (the "Bimodal Peak") resembling an acceleration or a sustained increase in the baseline FHR secondary to uterine hypertonus during induction of labour. Both uterine hyperstimulation (Figure 4) and hypertonus require an immediate action to rapidly re-oxygenate the fetus by removing the inciting agent (i.e., prostaglandins or oxytocin), and/or the use of tocolytics to avoid hypoxic-ischemic brain injury.

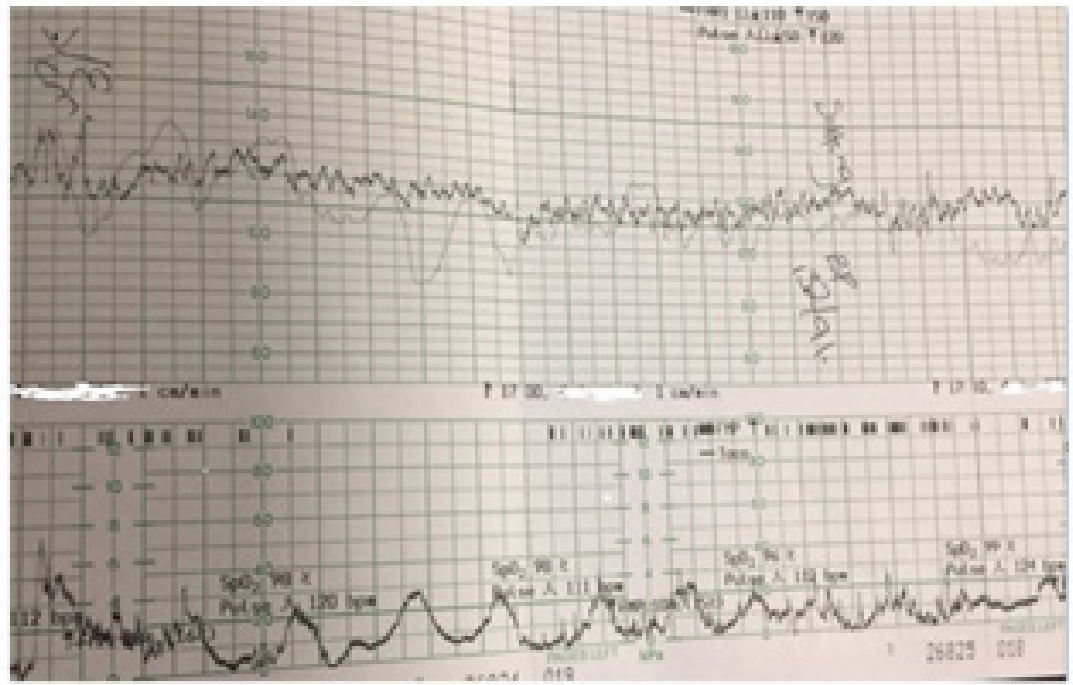

Figure 2: Spontaneous hyperstimulation with reduced variability and the "Poole Shark Teeth" Pattern commonly seen in concealed abruption.

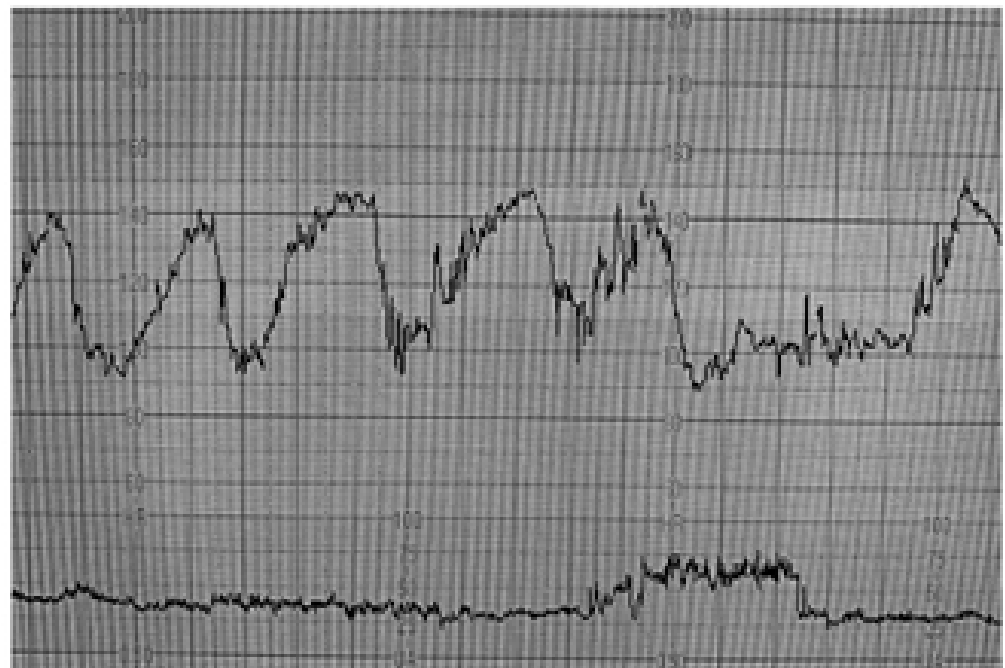

Figure 3: Uterine hypertonus with Fetal heart rate changes. 


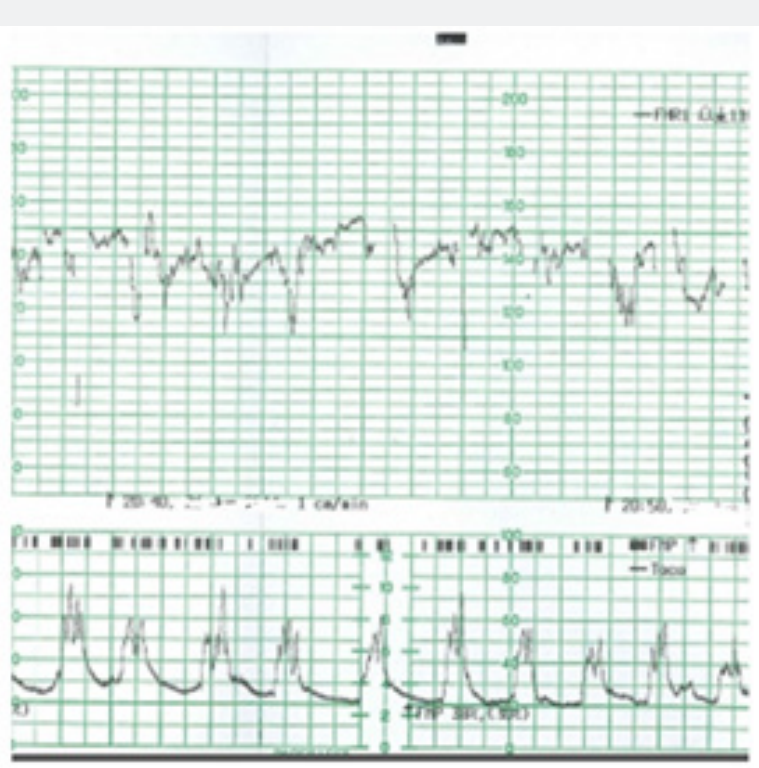

Figure 4: Hyperstimulation with Dinoprostone pessary in-situ.

\section{Use of tocolytics in uterine hyperstimulation}

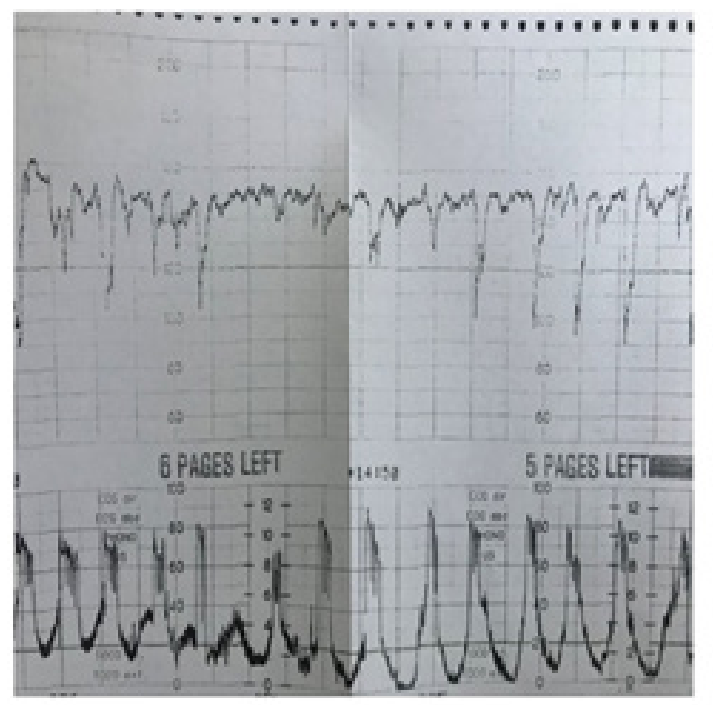

Figure 5: Uterine Tachysystole with CTG changes (hyperstimulation).

Uterine hyperstimulation (Figure 5) reduces the ability of a fetus to maintain central organ oxygenation. The use of acute tocolysis with various myometrial relaxants has become widespread in clinical practice based on the presumption that uterine relaxation improves uteroplacental blood flow and therefore fetal oxygenation [22]. The most used drugs for "acute" tocolysis are betamimetics. In a study comparing intravenous terbutaline with nitroglycerine, it was shown that terbutaline was an effective tocolytic with less impact on maternal blood pressure compared to nitroglycerine [23]. Although this study addressed the effect of terbutaline on fetal heart rate changes, it did not mention its effect on the uterine activity [23]. In a recent Cochrane Systematic Review, when a selective Beta 2 agonist was compared with no tocolytic agent, the number of abnormal fetal heart rate pattern was less in the tocolysis group, however, the effect on Apgar score was uncertain [24]. This review concluded that the clinical significance for some of the improvements in measures of fetal wellbeing with tocolytics was unclear as the sample 


\section{Global Journal of Reproductive Medicine}

sizes were too small to detect effects on neonatal morbidity, mortality, or serious adverse effects. An increase in cumulative uterine activity in the last 30 minutes of birth is associated with abnormal fetal heart pattern and poor neonatal outcomes [25]. Acute tocolysis should also be considered in any situation where the continuation of even normal uterine activity may worsen fetal oxygenation and has the potential to hasten the ongoing fetal compromise such as an umbilical cord prolapse [26]. Injudicious use of oxytocin was reported to be contributory factor in $68.5 \%$ of medico-legal claims [27], and the Each baby Counts Report by the Royal College of Obstetricians and Gynecologists (RCOG) has suggested that approximately $20 \%$ of poor intrapartum outcomes were due to "induction and augmentation issues" [28]. Moreover, a recent Confidential Enquiries into Maternal Deaths in the UK (MBRRACE, 2018) has reported that uterine hyperstimulation from induced labor was frequently identified among women who had died from hemorrhage and amniotic fluid embolism [29]. Therefore, there is an urgent need to understand and timely recognize excessive uterine activity during labor, instead of relying on illogical definitions proposed by some guidelines on CTG interpretation. After several years of confusion, the recent International consensus Guidelines on Physiological CTG interpretation developed by 34 CTG experts from 14 countries have correctly defined uterine hyperstimulation as "any increase in uterine activity (i.e., frequency, duration and strength or the basal tone) associated with FHR abnormalities [30].

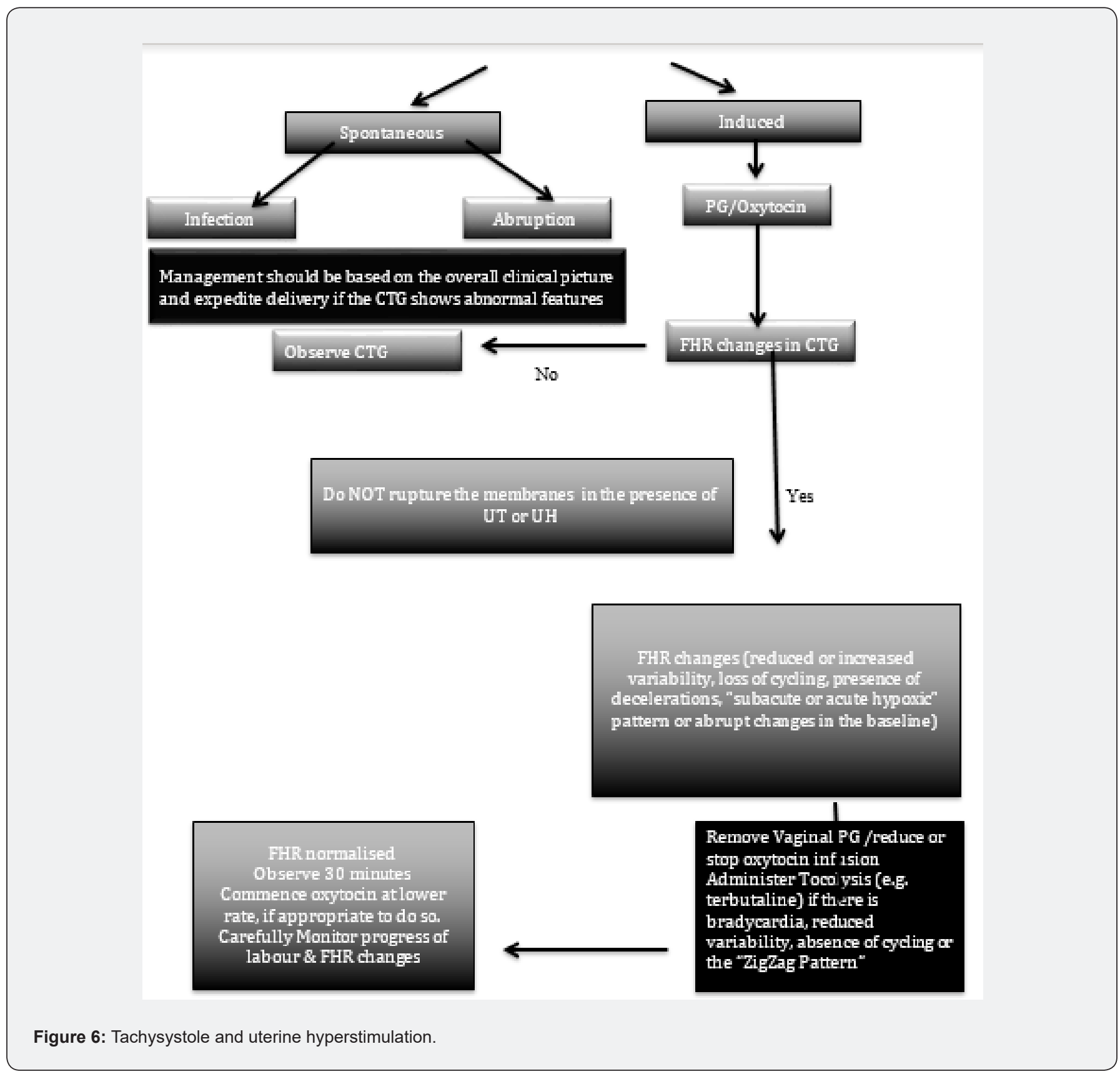




\section{Global Journal of Reproductive Medicine}

\section{Maternal complications of UT}

Complications to the mother secondary to UT or UH includes increased caesarean section rates, cervical laceration, uterine rupture in women with previous caesarean section, antepartum or postpartum hemorrhage as well as increased risk of amniotic fluid embolism. We have proposed an algorithm for management of uterine tachysystole and uterine hyperstimulation (Figure 6). This is aimed at considering the pathophysiology and managing excessive uterine activity appropriately including use of tocolysis and/or to consider delivery. A recent study on CTG changes in histopathologic ally confirmed cases of chorioamnionitis has concluded that hyperstimulation and myometrial irritability were observed in $>80 \%$ of cases, leading to an emergency caesarean sections in $95.4 \%$ of cases, predominantly due to abnormal FHR changes [31].

\section{Conclusion}

UT can occur in both spontaneous and induced labor. There is an urgent need for the clarification on definition and include the pathophysiology of uterine tachysystole and hyperstimulation, internationally. Midwives and obstetricians need to standardize the definitions of UT and UH based on the deeper understanding of underlying causes as well as the properties of uterine contractions (i.e., frequency, duration, strength, and the basal tone) to minimize the risks to the mother (i.e., uterine rupture or amniotic fluid embolism) and the fetus (hypoxic-ischemic injury). A fetus with reduced reserves such as IUGR and oligohydramnios or with RUPI may have less tolerance for UT or UH resulting in an increased risk of hypoxia and acidosis leading to hypoxic-ischemic injury or perinatal death. Therefore, it is vital to get the definition of hyperstimulation right to prevent avoidable intrapartum hypoxicischemic brain injury due to fetal compromise secondary to an ongoing excessive uterine activity.

\section{Declaration of Interest}

The authors report that they have no conflicts of interest to declare.

E. C. conducts several Master classes on CTG and fetal ECG in the UK, Europe, Asia. and Australia and is the co-organizer of the Intrapartum Fetal Surveillance Course at the Royal College of Obstetricians and Gynecologists (RCOG) He was a member of the Editorial Board for NHS e-learning on CTG and a Course lead for the Baby Lifeline CTG Masterclasses. Organisers of some of these Masterclasses have received sponsorships from Philips, Neoventa, Euroking, Huntleigh, K2 and other industry to support these Masterclasses. However, E.C. does not have any financial or managerial interests in any pharmaceutical or medical device industry. EC was one of the 3-member guideline development group which revised the international FIGO Guidelines on CTG in 2015, and he was on the Editorial Board which produced the first International Consensus Guidelines on Physiological CTG Interpretation

\section{Authorship and Contribution}

SS and EC conceived the idea. All authors declare substantial contributions to the acquisition, analysis, or interpretation of data and for revising it critically for important intellectual content. All authors approved the final version to be published and agree to be accountable for all aspects.

\section{References}

1. Reynolds AJ, Geary MP, Hayes BC (2020) Intrapartum uterine activity and neonatal outcomes: a systematic review. BMC pregnancy and childbirth 20(1): 532 .

2. Stewart RD, Bleich AT, Lo JY (2012) Defining uterine tachysystole: how much is too much? Am J Obstet Gynecol 207(290): e1-6.

3. Al-Hafez L, Chauhan SP (2019) Uterine tachysystole: a survey of CAOG members suggests persistent ambiguity. J Matern Fetal Neonatal Med 1: $1-6$.

4. Peebles DM, Spencer JAD, Edwards AD, Wyatt JS, Reynolds EOR, et al. (1994) Relation between frequency of uterine contractions and human fetal cerebral oxygen saturation studied during labour by near infrared spectroscopy. BJOG: An International Journal of Obstetrics \& Gynaecology 101: 44-48.

5. Stephen E Gee, Heather A Frey (2020) Contractions: Traditional concepts and their role in modern obstetrics. Seminars in Perinatology 44(2): 151218.

6. National Institute for Health and Care Excellence (2017) Intrapartum care

7. Ahmed AI, Zhu L, Aldhaheri S, Sakr S, Minkoff H, et al. (2016) Uterine tachysystole in spontaneous labor at term. J Matern Fetal Neonatal Med 29(20): 3335-3339.

8. Heinonen PK, Kajan M, Saarikoski S (1986) Cardiotocographic findings in abruptio placentae. European Journal of Obstetrics and Gynecology 23(1): 75-78.

9. Vlemminx MW, De Lau H, Oei SG (2017) Tocogram characteristics of uterine rupture: a systematic review. Arch Gynecol Obstet 295(1): 1726.

10. Louden E, Marcotte M, Mehlman C, Lippert W, Huang B, et al. (2018) Risk Factors for Brachial Plexus Birth Injury. Children 5: 46.

11. Alfirevic Z, Keeney E, Dowswell T (2015) Labor induction with prostaglandins: a systematic review and network meta-analysis. BM] (Clinical research ed) 350: h217.

12. Ziyauddin F, Hakim S, Beriwal S (2013) The Transcervical Foley Catheter Versus the Vaginal Prostaglandin E2 Gel in the Induction of Labour in a Previous One Caesarean Section-A Clinical Study. Journal of Clinical and Diagnostic Research 7(1): 140-143.

13. Mozurkewich EL, Chilimigras JL, Berman DR (2011) Methods of induction of labour: a systematic review. BMC Pregnancy Childbirth

14. Kelly AJ, Malik S, Smith L, Kavanagh J, Thomas J (2010) Vaginal prostaglandin (PGE2 and PGF2a) for induction of labour at term. Cochrane Database of Systematic Reviews.

15. Boulvain M, Kelly AJ, Irion O (2009) Intracervical prostaglandins for induction of labour. Cochrane Database of Systematic Reviews.

16. Maggi C, Mazzoni G, Gerosa V, Fratelli N, Prefumo F, et al. (2020) Labor induction with misoprostol vaginal insert compared with dinoprostone vaginal insert. Acta Obstetricia et Gynecologica Scandinavica 98(10): 1268.

17. Alfirevic Z, Kelly AJ, Dowswell T (2010) Intravenous oxytocin alone for cervical ripening and induction of labour. Cochrane Database of Systematic Reviews 2: 2. 
18. Heinemann J, Gillen G, Ramos LS, Kaunitz AM (2008) Do mechanical methods of cervical ripening increase infectious morbidity? A systematic review. American Journal of Obstetrics \& Gynecology 199(2): 177-187.

19. Verspyck E, Sentilhes L (2008) Abnormal fetal heart rate patterns associated with different labor managements and intrauterine resuscitation techniques. Journal de gynecologie, obstetrique et biologie de la reproduction 37 Suppl 1: S56-64.

20. Gracia Perez-Bonfils A, Vigneswaran K, Cuadras D, Chandraharan E (2019) Does the saltatory pattern on cardiotocograph (CTG) trace really exist? The ZigZag pattern as an alternative definition and its correlation with perinatal outcomes. J Matern Fetal Neonatal Med 13: $1-9$.

21. Tarvonen M, Hovi P, Sainio S, Vuorela P, Andersson S (2020) Intrapartum zigzag pattern of fetal heart rate is an early sign of fetal hypoxia: A large obstetric retrospective cohort study. Acta Obstet Gynecol Scand 27.

22. Kulier R, Hofmeyr GJ (2000) Tocolytics for suspected intrapartum fetal distress. Cochrane Database Syst Rev 2.

23. Pullen KM, Riley ET, Waller SA, Taylor L, Caughey AB, et al. (2007) Randomized comparison of intravenous terbutaline vs nitroglycerin for acute intrapartum fetal resuscitation. American Journal of Obstetrics and Gynecology 197(4): 414.

24. Leathersich SJ, Vogel JP, Tran TS, Hofmeyr GJ (2018) Acute tocolysis for uterine tachysystole or suspected fetal distress. Cochrane Database of Systematic Reviews 7.
25. O Dickinson, V Lowe, E Chandraharan (2012) Does increased cumulative uterine activity (frequency and duration of uterine contractions) during the last 30 minutes prior to delivery affect the incidence of pathological decelerations and fetal outcome? an observational study. International Journal of Gynecology \& Obstetrics S531-S867.

26. Chandraharan E, Arulkumaran S (2016) Acute tocolysis. Curr Opin Obstet Gynecol 17(2): 151-156.

27. Jonsson M, Nordén SL, Hanson U (2007) Analysis of malpractice claims with a focus on oxytocin use in labour. Acta Obstet Gynecol Scand 86(3): 315-319.

28. (2018) Royal College of Obstetricians and Gynaecologists. Each Baby Counts: 2018 Progress Report. London: RCOG.

29. Knight M, Nair M, Tuffnell D, Shakespeare J, Kenyon S, et al. (Eds.), On behalf of MBRRACE-UK. Saving Lives, Improving Mothers' Care - Lessons learned to inform maternity care from the UK and Ireland Confidential Enquiries into Maternal Deaths and Morbidity.

30. Sukumaran S, Pereira V, Mallur S, Chandraharan E (2021) Cardiotocograph (CTG) changes and maternal and neonatal outcomes in chorioamnionitis and/or funisitis confirmed on histopathology. Eur J Obstet Gynecol Reprod Biol 260: 183-188.

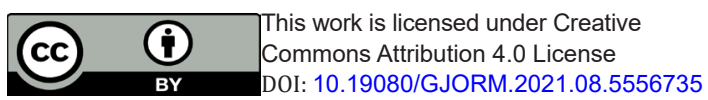

